\title{
Eosinophilic cystitis presenting as a bladder mass in an 11-year-old girl
}

Elsa Bey ${ }^{1,2}$; Youssef Teklali ${ }^{1}$; Pierre-Yves Rabattu ${ }^{1}$; Simon Grandjean Lapierre ${ }^{3}$; C. Piolat ${ }^{1}$ ${ }^{1}$ Department of Pediatric surgery, University Hospital of Grenoble, France; ${ }^{2}$ Department of Urology and Renal Transplantation, University Hospital of Grenoble, France; ${ }^{3}$ Department of Microbiology and Infectious Diseases, Centre hospitalier de l’Université de Montréal, Montreal, QC, Canada

Cite as: Can Urol Assoc J 2017; Epub ahead of print. http://dx.doi.org/10.5489/cuaj.4513

Published online November 1, 2017

\section{Introduction}

We report the case of an 11-year-old girl who presented with acute urinary retention and microscopic hematuria. Pelvic imaging revealed a bladder mass for which sarcoma was initially suspected. Surgical biopsies revealed a massive eosinophilic infiltration of the submucosa confirming a diagnosis of eosinophilic cystitis. Clinical evolution was favourable without treatment. Eosinophilic cystitis prognosis is usually benign, but rare severe complications, such as complete bladder fibrosis requiring bladder augmentation or cystectomy, may occur. 


\section{Case report}

Eosinophilic cystitis was first described by Brown in $1960^{1}$ and about fifty pediatric cases have been described in the literature since. We report the case of a 11-year old girl with eosinophilic cystitis presenting as painless acute urinary retention associated with microscopic hematuria and favorable evolution without treatment.

The patient was referred to Grenoble University Hospital's emergency room by her primary care physician for a 2-days history of supra-pubic pain, dysuria and microscopic hematuria. She had no past medical history or allergies. She did not report any regular or sporadic medication intake, past tropical areas travel history or familial atopy manifestations.

Symptoms were associated with acute fatigue but she had no history of weight loss, diarrhea, fever or rash. Clinical examination revealed an isolated painless acute urinary retention without hepato-splenomegaly, fever or cutaneous manifestations. Urinary labstix showed microscopic hematuria, automated urinalysis 10000 red blood cells/mL, few white blood cells and no eosinophiluria. Complete blood count, blood eosinophils, C-Reactive Protein $(<3 \mathrm{mg} / \mathrm{L})$ and renal function were normal. Abdominal ultrasonography revealed a trigonal bladder mass of 10x10 mm with secondary urinary retention of 700cc without upper urinary tract dilatation.

Urinary drainage by Foley catheter was performed and the patient was hospitalized. Alfa-foetoprotein, ß-hCG and LDH bladder sarcoma markers were normal. Pelvic magnetic resonance imaging (MRI) showed a 10x10 mm bladder mass with a strong gadoliniumenhancing profile. Primary suspected diagnosis was rhabdomyosarcoma (Fig. 1). A positron emission tomography was performed and revealed intense fixation at the same anatomical site. No suspicious distant fixation foci were observed on this exam.

A cystoscopy under general anesthesia was performed and showed a flat edematous hyper vascularized lesion with unclear borders localized at the bladder neck. Pathology colorations on biopsy tissue revealed a non-specific inflammatory infiltrate not allowing definitive diagnosis. Since rhabdomyosarcoma was suspected, close follow-up hospitalization with diagnostic laparotomy was scheduled 7 days later.

A short supra-pubic Pfannenstiel incision was performed, followed by a transvesical approach under cystoscopic control. The surgery was completed through pneumovesicoscopic approach allowing good and direct visualization of the lesion (Fig. 2). The pneumovesicoscopic approach revealed an edematous and hypervascularized lesion implicating the whole bladder neck. No induration or ulceration were seen. Six macrobiopsies including the whole macroscopic lesion were taken. A bladder catheter was maintained until macroscopic hematuria resolution on day 4 after surgery. A clamped suprapubic security catheter was also initially left in place. Patient's urinary function recovered completely without any post-void residue. Macroscopic biopsies showed an important eosinophilic infiltrate of the chorion and submucosa typical of eosinophilic cystitis (Fig. 3). Clinical evolution was favorable without specific treatment no recurrence or complications were observed up to 7 months of follow-up. Complementary infectious work-up including Toxocara gondii serology came back negative. 


\section{Discussion}

Eosinophilic cystitis is a rare and exceptional condition respectively in adults and children. The median age at diagnosis in the pediatric population is 6.5 years old ${ }^{2}$, with a sex ratio of $3: 1$ over-representing males ${ }^{3,4}$ : this case is therefore uncommon compared to the usual epidemiology. The clinical presentation is very diverse, usually associating dysuria, microscopic hematuria and pollakiuria ${ }^{3,4}$. Active urinary tract infection is present in nearly half of the cases ${ }^{5}$ at time of diagnosis. Eosinophilic cystitis has been described in very diverse clinical contexts such as post-operative, following a viral or parasitic infection, after intravesical instillation of mitomycin $\mathrm{C}$ and during acute kidney stone disease amongst others. It is therefore difficult to establish clear causal or risk factors for this condition ${ }^{2}$. Association with other atopic features was only found in about $33 \%$ of patients in a recent review ${ }^{4}$. An association with Toxocara gondii infection was described by Perlmutter and al. therefore justifying a systematic serology ${ }^{6}$. Our patient did not present any predisposing conditions or risk factors. About $40 \%$ of cases present with eosinophilia, which is an inconsistent finding such as eosinophiluria ${ }^{7,8}$. The clinical and radiological presentation is frequently that of a bladder mass, resulting in initial high suspicion of in situ carcinoma in adults and rhabdomyosarcoma in children ${ }^{9,10}$. Widespread thickening of the whole bladder wall and unilateral ureteral dilatation, present in one third of cases, are other possible manifestations ${ }^{7}$. Independently of the initial presentation, lesions are usually completely self-resolving. The initial malignancy suspicion often leads to a quick cystoscopy and biopsies which are mandatory to establish the diagnosis ${ }^{3}$. Typical endoscopic aspects include submucosal hemorrhage, ulceration, edema and hyper-vascularization ${ }^{8}$, which correspond to our patient's findings [Fig. 2]. Proposed treatment algorithm by Ozdoğan in 2014 includes non-steroidal anti-inflammatories and anti-histaminics as first line agents, followed by corticosteroids in case of insufficient improvement after 2 weeks of treatment ${ }^{11}$. Alternatively, a "watchful waiting” approach including clinical and ultrasonographic surveillance was proposed by Itano $^{12}$. In this alternative algorithm, pharmaceutical treatment is reserved for cases in which symptoms persist after 2 weeks and removal of potential antigenic stimuli (treatment of active urinary tract infection, antigen eviction if atopy is reported, treatment of toxocarosis) ${ }^{12}$. To our knowledge, no malignant evolution has been reported but the frequency of recurrences and potential chronicity justifies close follow-up for at least one year after complete resolution ${ }^{7,8}$. Corticosteroids seem to be very efficient allowing complete and sustained remission in 2 weeks compared to 8 to 10 weeks remission delays without treatment ${ }^{8}$. Immunosuppressive therapies such as cyclosporine have been proposed in exceptional cases of insufficient improvement under corticosteroids ${ }^{5,11}$. Ozdoğan proposes a regular follow-up including clinical examination, ultrasonography and control of initially elevated plasmatic eosinophils count. In cases of persistent ultrasonographic abnormalities under corticosteroid therapy a control cystoscopy should also be performed. Several publications reported unfavorable evolution such as complete bladder fibrosis with reduced compliance requiring aggressive surgical treatments such as bladder augmentation or cystectomy ${ }^{5,7,12}$. 


\section{Conclusion}

Eosinophilic cystitis is a difficult to recognize and probably under-diagnosed condition whose pathogenesis is still poorly understood. This case expands our knowledge and the existing literature with cystoscopy, radiology and pathology visual material that may help urologic surgery, radiology and pathology teams when confronted to such atypical findings. This case also reinforces the need for endoscopic biopsies to confirm diagnosis and initiate optimal management of this rare pediatric pathology which has good prognosis when optimally managed. 


\section{References}

1. Brown EW. Eosinophilic granuloma of the bladder. J Urol. 1960;83:665-668.

2. Cohen J, Letavernier B, Garel C, Boubnova J, Boudjemaa S, Bensman A. Cystite à éosinophiles chez l'enfant. Arch Pédiatrie. 2011;18(5):550-552. doi:10.1016/j.arcped.2011.02.021.

3. van den Ouden D. Diagnosis and management of eosinophilic cystitis: a pooled analysis of 135 cases. Eur Urol. 2000;37(4):386-394. doi:20183.

4. Sparks S, Kaplan A, DeCambre M, Kaplan G, Holmes N. Eosinophilic cystitis in the pediatric population: A case series and review of the literature. J Pediatr Urol. 2013;9(6, Part A):738-744. doi:10.1016/j.jpurol.2012.11.004.

5. THOMPSON RH, DICKS D, KRAMER SA. CLINICAL MANIFESTATIONS AND FUNCTIONAL OUTCOMES IN CHILDREN WITH EOSINOPHILIC CYSTITIS. $J$ Urol. 2005;174(6):2347-2349. doi:10.1097/01.ju.0000180423.06285.72.

6. Perlmutter AD, Edlow JB, Kevy SV. Toxocara antibodies in eosinophilic cystitis. $J$ Pediatr. 1968;73(3):340-344. doi:10.1016/S0022-3476(68)80110-6.

7. Slama A, Khouni H, Sriha B, Brini K, Sorba NB, Mosbah AT. Fibrose vésicale due à une cystite à éosinophiles. Ann Urol. 2003;37(5):272-274. doi:10.1016/S00034401(03)00054-8.

8. Ladocsi LT, Sullivan B, Hanna MK. Eosinophilic granulomatous cystitis in children. Urology. 1995;46(5):732-735. doi:10.1016/S0090-4295(99)80313-6.

9. NETTO JMB, PÉREZ LM, KELLY DR, JOSEPH DB. PEDIATRIC INFLAMMATORY BLADDER TUMORS: MYOFIBROBLASTIC AND EOSINOPHILIC SUBTYPES. J Urol. 1999;162(4):1424-1429. doi:10.1016/S00225347(05)68329-2.

10. THOMAS JC, ROSS JH. EOSINOPHILIC CYSTITIS IN A CHILD PRESENTING WITH A BLADDER MASS. J Urol. 2004;171(4):1654-1655. doi:10.1097/01.ju.0000115722.65503.d9.

11. Ozdoğan EB, Arslansoyu Çamlar S, Bilen S, et al. An unusual cause of terminal hematuria in a child: Eosinophilic cystitis. Can Urol Assoc J J Assoc Urol Can. 2014;8(11-12):E867-871. doi:10.5489/cuaj.2173.

12. ITANO NMB, MALEK RS. EOSINOPHILIC CYSTITIS IN ADULTS. J Urol. 2001;165(3):805-807. doi:10.1016/S0022-5347(05)66531-7. 


\section{Figures and Tables}

Fig. 1. Pelvic MRI. T2 injected sequence showing a gadolinium-enhancing bladder mass of $10.3 \mathrm{~mm}$ with urinary retention.

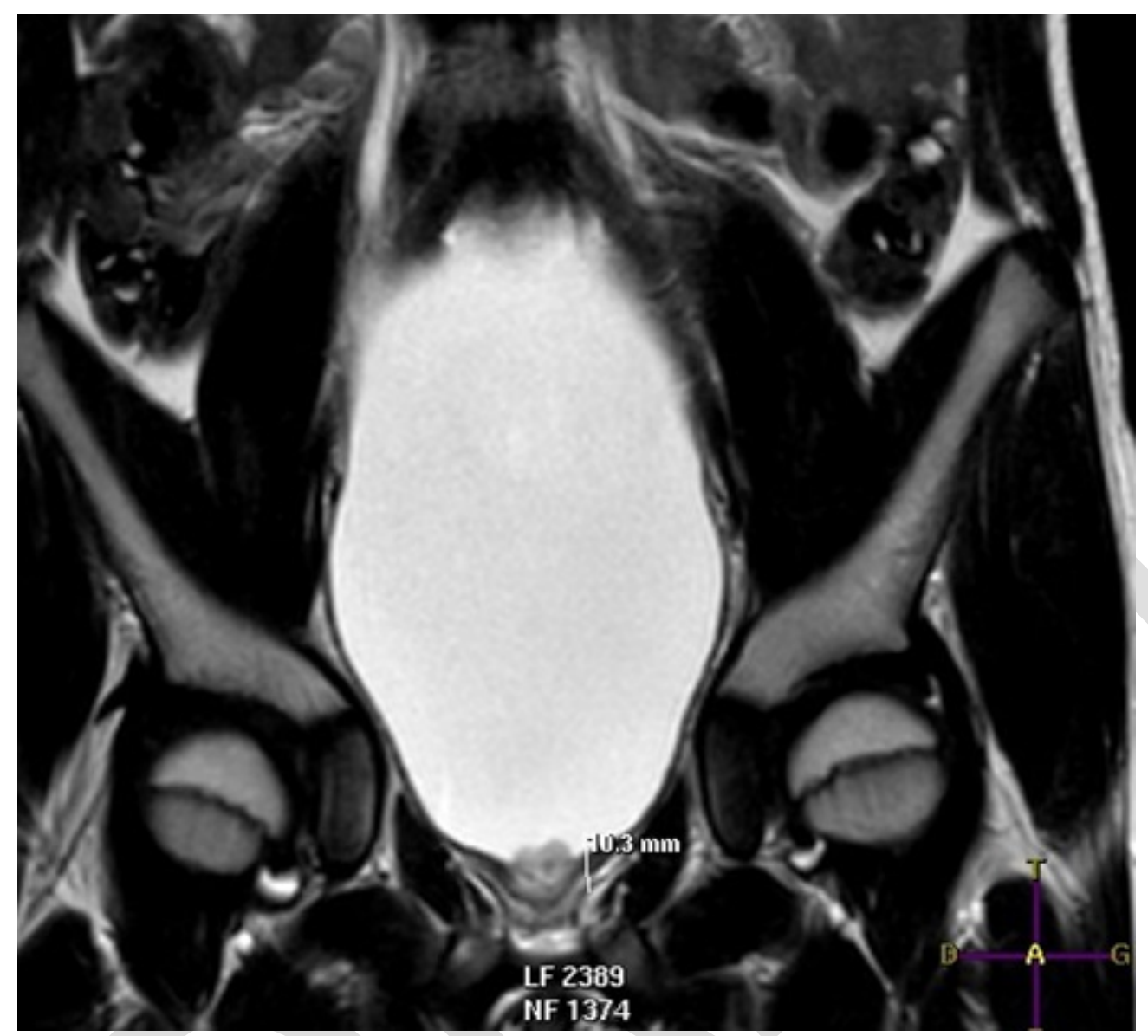


Fig. 2. Pneumo-vesicoscopy of the bladder neck showing a typical aspect of eosinophilic cystitis, with hyper-vascularized inflammatory edema in "medusa's head."

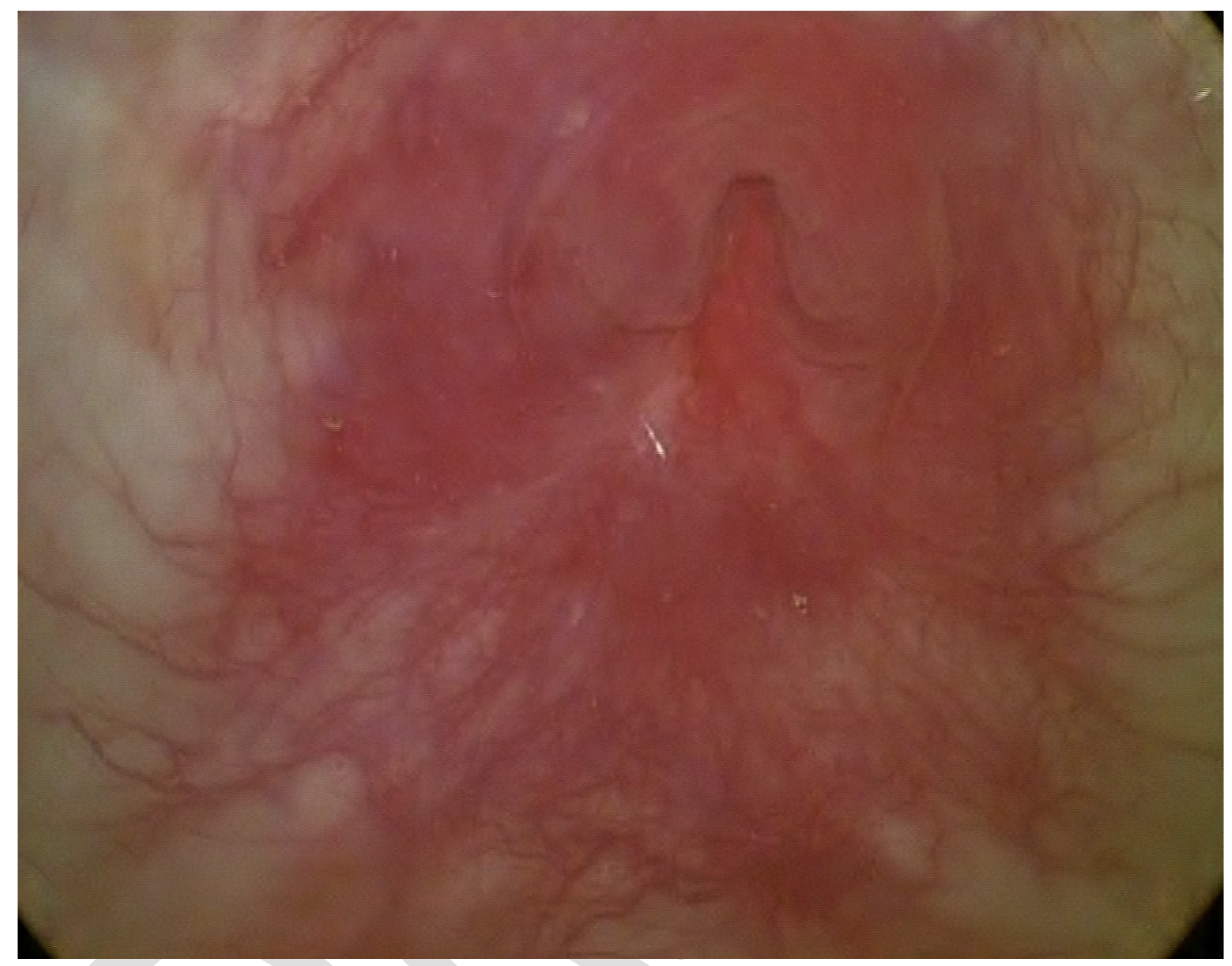


Fig. 3. Bladder mass biopsy showing important eosinophilic infiltrate of the submucosa on H\&E coloration (color printing required).
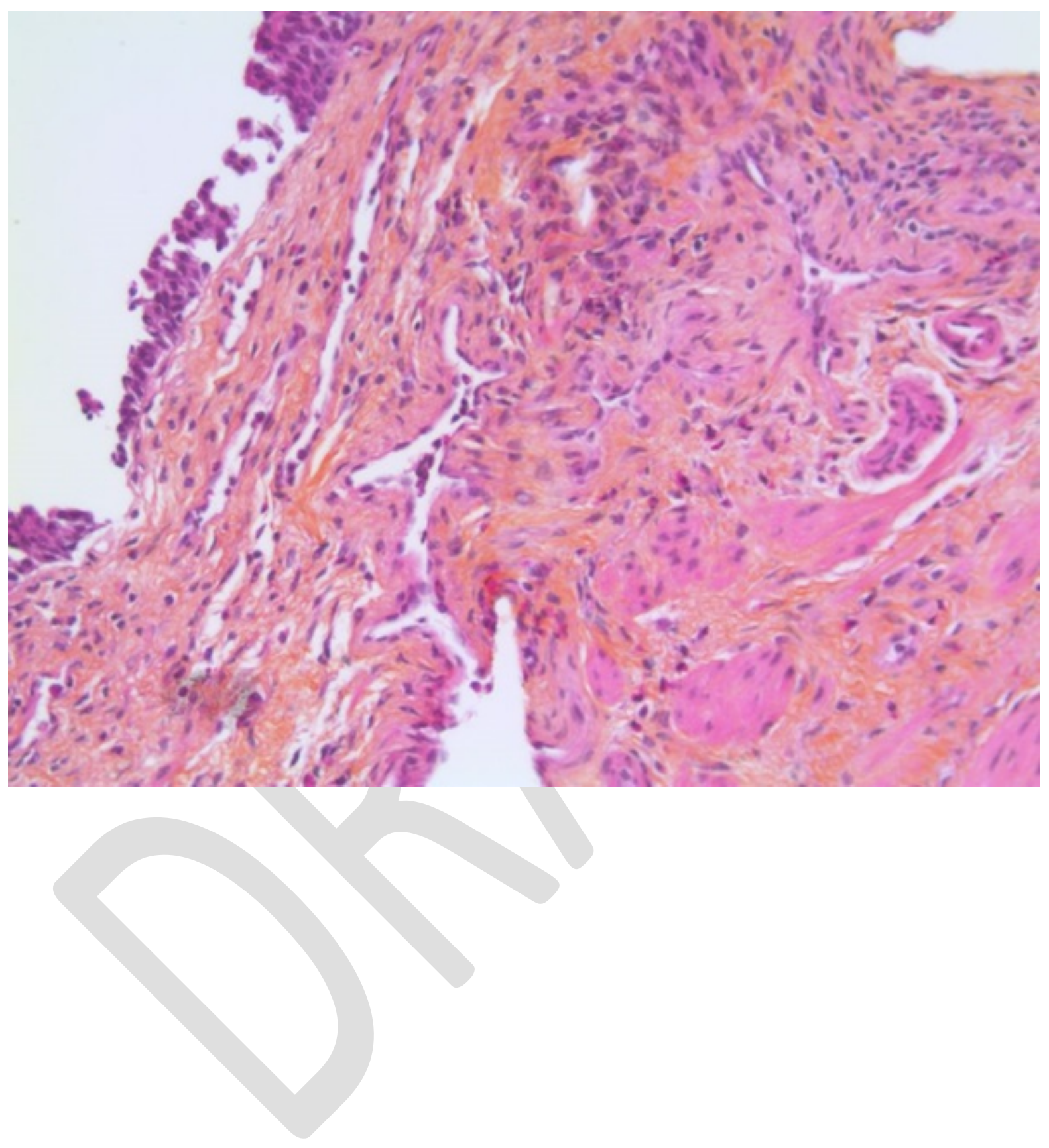